Authors' reply: Balanzá-Martinez et al made a detailed review of our article ${ }^{1}$ and also provided several suggestions for future research.

Although we agree with the authors that the conclusions of our article are by no means definitive, we disagree with them on the following important issues.

First, Balanzá-Martinez et al suggest that our meta-analysis 'more likely overestimates the potential for cognitive improvement' as compared with other reviews (the authors cite as an example the review by Rund $^{2}$ ). On the contrary, we think that the comparison of results in individuals with schizophrenia with controls, which is an original feature of our review, has a sobering effect as it points out that 'practice [is] more likely than cognitive remediation to account for most of the improvements observed'. On the other hand, reviews (such as the one cited earlier) in which performances in people with schizophrenia are not compared with those of controls could mistake improvement in results for improvement in cognitive abilities.

Second, commenting on the methods we used in our metaanalysis, Balanzá-Martinez et al criticise three of our options: (a) the inclusion of studies with a test-retest duration under 1 year; (b) the inclusion of open trials (especially clozapine trials); and (c) the fact that we did not differentiate between 'patients with first-episode or chronic schizophrenia and geriatric patients' They suggest therefore that it would be better to limit the analyses to a subset of the available data assuming, without formally testing, that some of the studies' characteristics significantly influence results.

At the time we made our analysis, there were only 11 studies with more than a year test-retest interval and only 4 reported data for a control group. It was not possible to limit our analysis only to this subset of studies, let alone further exclude studies or separately analyse subgroups of studies.

Instead, we chose to include all methodologically sound studies and test the role of potentially confounding variables (including test-retest interval). By using this method, we limited the loss of important available information. It is our belief that we could not gain more insight from fewer data.

As we report in our article, only 2 out of the 17 variables tested showed a larger improvement in studies with shorter test-retest intervals. Thus, for the vast majority of variables, excluding studies with shorter test-retest intervals would lead to an unjustified loss of information.

Third, Balanzá-Martinez et al consider that their suggestions may lead to results 'more helpful for clinicians, patients and caregivers'. We think that limiting the analysis to studies with large test-retest intervals (which usually have high attrition rates) or excluding the more naturalistic, open trials would achieve just the contrary.

In conclusion, although we agree with Balanzá-Martinez et al that the conclusions of our meta-analysis are not definitive, we also consider that to improve our knowledge on the subject we need new data, not new analyses, in subsets of (the same) data.

1 Szöke A, Trandafir A, Dupont ME, Méary A, Schürhoff F, Leboyer M. Longitudinal studies of cognition in schizophrenia: meta-analysis. Br J Psychiatry 2008; 192: 248-57.

2 Rund BR. A review of longitudinal studies of cognitive functions in schizophrenia patients. Schizophr Bull 1989; 24: 425-35.

Andrei Szöke, Franck Schürhoff, Marion Leboyer, AP-HP, GHU 'ChenevierMondor', Pôle de Psychiatrie, 94000, Créteil, France; Université Paris XII, Faculte de Médecine, 9400, Créteil, and INSERM U955, Créteil, France. Email:

andrei.szoke@ach.aphp.fr

doi: 10.1192/bjp.195.1.85

\section{Neural correlates of formal thought disorder}

Horn et al investigate a very important, somewhat underexplored area of neural correlates of schizophrenic speech disturbance. Given the probability of underlying deficits in contextual integration and theory of mind, formal thought disorder yields a fertile ground for structural and functional connectivity analysis in schizophrenia. Although the use of hitherto unused techniques such as resting perfusion scan to study formal thought disorder must be lauded, the results of this preliminary study must be treated with caution for various reasons.

The composite score of the Scale for the Assessment of Thought, Language and Communication (TLC) has been used as a measure of severity of formal thought disorder. The authors have administered the scale $45 \mathrm{~min}$ before the scanning procedure for each participant. It is widely perceived that uncontrolled generation of thought is required to reliably measure formal thought disorder in schizophrenia. The TLC itself lacks a standardised practical method of eliciting such thought flow in contrast to some recently developed instruments. ${ }^{2}$ The crosssectional use of the TLC to measure formal thought disorder severity must be treated with prudence.

The authors extract components from the Positive and Negative Syndrome Scale (PANSS) using factor analysis and demonstrate that none of these components correlate with formal thought disorder severity as measured by the TLC total score. The validity of factor analysis in such a small sample is questionable and not in synchrony with available factorial structures of PANSS. ${ }^{3}$ As a result, all principal components extracted were from negative symptoms in PANSS (except the conceptual disorganisation item, which was rightly excluded from further analysis). Consequently, the results only show a lack of correlation between severity of formal thought disorder and negative symptoms as measured by PANSS. Findings from the magnetic resonance imaging may still be explained by positive symptoms alone and not by formal thought disorder. Lastly, the pervasive issue of sample size in neuroimaging studies becomes more prominent when correlation analyses are attempted in whole brain analyses.

1 Horn H, Federspiel A, Wirth M, Müller TJ, Wiest R, Wang J-J, et al. Structural and metabolic changes in language areas linked to formal thought disorder. Br J Psychiatry 2009; 194: 130-8.

2 Liddle PF, Ngan ETC, Caissie SL, Anderson CM, Bates AT, Quested DJ, et al. Thought and Language Index: an instrument for assessing thought and language in schizophrenia. Br J Psychiatry 2002; 181: 326-30.

3 Fitzgerald PB, de Castella AR, Brewer K, Filia K, Collins J, Davey P, et al. A confirmatory factor analytic evaluation of the pentagonal PANSS model. Schizophr Res 2003; 61: 97-104.

Lena Palaniyappan, Division of Psychiatry, Newcastle University, Newcastle upon Tyne NE1 4LP, UK. Email: Lena.Palaniyappan@ncl.ac.uk

doi: 10.1192/bjp.195.1.85a

Authors' reply: Palaniyappan suggests using the Thought and Language Index (TLI) instead of the TLC in order to quantify formal thought disorder. The TLI, he argues, would have the advantage over the TLC of a standardised method of eliciting thought flow. Unfortunately, the TLI was established after the start of our study, ${ }^{1}$ whereas the TLC was an established instrument that has been successfully used in numerous studies as a reliable instrument to quantify formal thought disorder. ${ }^{2}$ We agree that future studies might benefit from the application of the more standardised TLI. However, the distribution of the severity of formal thought disorder in the patient group should not change 
substantially just by changing from the TLC to the TLI. Therefore, we regard our results as reliable.

Furthermore, Palaniyappan criticises the factor analysis of the PANSS in the small patient samples used in neuroimaging studies. He is right that a factor analytic approach in such small samples is critical if the patients group is randomly selected. In order to be able to study formal thought disorder with neuroimaging techniques, we recruited a specifically selected patient sample, which mainly differs in formal thought disorder severity and barely in other psychopathological categories. To obtain such a selected sample, the recruitment took years. Patients were matched as closely as possible for all other psychopathology traits in addition to the items of formal thought disorder of PANSS. The factor analysis and the correlation of the factors with the severity of formal thought disorder were only used to document this special patient selection. Therefore, it is no surprise that the factors of our factor analysis do not match the factors of unselected samples of patients with schizophrenia. ${ }^{3}$ To study specific psychopathological phenomena like formal thought disorder by means of neuroimaging, such a specific selection of patients is necessary to extract reliable results. In our patient group we did not observe any significant correlation between formal thought disorder and (a) positive symptoms (without PANSS item P2): $r=0.39, P=0.19$; and (b) negative symptoms (without PANSS item N5): $r=0.04, P=0.90$. We therefore can conclude that our results are due to positive or negative symptoms in general.

Concerning the issue of the sample size in neuroimaging studies, our results were corrected for multiple comparisons. This approach is a common and accepted way to handle this problem in neuroimaging studies in general.

1 Liddle PF, Ngan ETC, Caissie SL, Anderson CM, Bates AT, Quested DJ, et al. Thought and Language Index: an instrument for assessing thought and language in schizophrenia. Br J Psychiatry 2002; 181: 326-30.

2 Andreasen NC, Grove W. Thought, language and communication in schizophrenia: diagnosis and prognosis. Schizophr Bull 1986; 12: 348-59.

3 Fitzgerald PB, de Castella AR, Brewer K, Filia K, Collins J, Davey P, et al. A confirmatory factor analytic evaluation of the pentagonal PANSS model. Schizophr Res 2003; 61: 97-104.

Helge Horn, University Hospital of Psychiatry, University of Bern, Switzerland. Email: horn@puk.unibe.ch; Andrea Federspiel, University Hospital of Psychiatry, University of Bern, Switzerland

doi: 10.1192/bjp.195.1.85b

\section{Public attitudes towards mental illness}

It is tempting to accept the conclusion from the paper by Mehta et $a l^{1}$ that the Scottish anti-stigma campaign 'see me' has successfully influenced public attitudes north of the border. However, it is not clear that this is an appropriate conclusion from the data they present.

They describe random sampling techniques whereby 2000 adults representative of the UK population were surveyed. One presumes that this would give rise to cohorts in England which were roughly ten times larger than those in Scotland. Comparing the year 2000 against 2003, they observed a deterioration for 17/25 stigma questionnaire items in England against only 4/25 in Scotland, and concluded that Scotland's dubious distinction of having done less badly suggested that 'see me' had been effective. Can they assure us that this difference did not arise simply because the much larger samples in England would be more likely to show a statistically significant difference than smaller Scottish samples?
1 Mehta N, Kassam A, Leese M, Butler G, Thornicroft G. Public attitudes towards people with mental illness in England and Scotland, 1994-2003. Br J Psychiatry 2009; 194: 278-84.

John M. Eagles, Royal Cornhill Hospital, Cornhill Road, Aberdeen AB25 2ZH, Scotland. Email: john.eagles@nhs.net

doi: 10.1192/bjp.195.1.86

Authors' reply: Professor Eagles writes to ask whether our conclusion from the paper is that the Scottish 'see me' anti-stigma campaign has positively influenced public attitudes about mental illness in Scotland. He suggests that this conclusion may be inappropriate because the populations in Scotland and England produce different sample sizes, given that the population of England is roughly ten times that of Scotland. We are very grateful to Professor Eagles for his comments as they allow us to provide some more information on these surveys than we could include in the original paper. ${ }^{1}$

As he rightly says, direct evidence of the position in Scotland vis-à-vis England is not provided by comparison of only those significant changes within the two sites. We had hoped to pursue this question further with analysis of future surveys, which would have given us power to make the comparisons between Scotland and England adequately, but unfortunately the wording of the Scottish survey has been changed so this will not be possible. The existing data-sets do, however, show limited evidence in favour of Scotland when comparing their respective mean changes from immediately pre- to post-campaign periods (2000 v. 2003). Of the 25 items, 6 differed between the sites at a nominal 0.1 significance level. One item (26: fear of downgrading residential areas) favoured England at $P=0.05$. The others favoured Scotland: items 7-9 at $P=0.1$ (to do with tolerance), and items 10 and 13 (the need to spend money and care for people with mental illness) at $P=0.05$. As we stated in our paper, the evidence may be consistent with an early positive effect of 'see me', but this possible association requires further investigation, although we accept that it is far from conclusive and needs further verification.

1 Mehta N, Kassam A, Leese M, Butler G, Thornicroft G. Public attitudes towards people with mental illness in England and Scotland, 1994-2003. Br J Psychiatry 2009; 194: 278-84.

Nisha Mehta, Health Service and Population Health Research Department, Institute of Psychiatry, King's College London, Box PO29 De Crespigny Park, London SE5 8AF, UK. Email: nisha.s.mehta@kcl.ac.uk; Aliya Kassam, Morven Leese, Georgia Butler, Graham Thornicroft, Health Service and Population Health Research Department, Institute of Psychiatry, King's College London, UK

doi: 10.1192/bjp.195.1.86a

\section{Is there core diffusion tensor imaging pathology in schizophrenia?}

Kanaan et al ${ }^{1}$ reported widespread abnormalities in white matter in 76 patients with schizophrenia compared with 76 healthy controls. A secondary analysis of 45 patients showed mean extracted fractional anisotropy scores to be unrelated to illness duration and duration of antipsychotic treatment. We wish to make two comments.

First, their main hypothesis that they would reconcile inconsistencies in the literature is a worthy, but elusive, goal. The problem of nosological heterogeneity 'afflicts' not only the definition of schizophrenia, but also the interpretation of fractional anisotropy localisation. Fractional anisotropy score localities are commonly cited in terms of grey or white matter terminology. Given such heterogeneity, it would suffice to adopt 